Published in final edited form as:

Arch Osteoporos. ; 12(1): 91. doi:10.1007/s11657-017-0389-1.

\title{
Comparison of fracture risk assessment tools in older men without prior hip or spine fracture: the MrOS Study
}

Margaret L. Gourlay, MD, MPH${ }^{1}$, Victor S. Ritter, $\mathrm{MS}^{2}$, Jason P. Fine, $\mathrm{ScD}^{2}$, Robert A. Overman, PhD, MPH ${ }^{3}$, John T. Schousboe, MD, $\mathrm{PhD}^{4,5}$, Peggy M. Cawthon, PhD, $\mathrm{MPH}^{6}$, Eric S. Orwoll, $\mathrm{MD}^{7}$, Tuan V. Nguyen, $\mathrm{PhD}^{8}$, Nancy E. Lane, $\mathrm{MD}^{9}$, Steven R. Cummings, $\mathrm{MD}^{6}$, Deborah M. Kado, MD, MS ${ }^{10,11}$, Jodi A. Lapidus, PhD ${ }^{12}$, Susan J. Diem, MD, MPH ${ }^{13,14}$, Kristine E. Ensrud, MD, MPH ${ }^{13,14,15}$, and for the Osteoporotic Fractures in Men (MrOS) Study Group

${ }_{1}^{1}$ Department of Family Medicine, University of North Carolina, Chapel Hill, North Carolina

${ }^{2}$ Department of Biostatistics, University of North Carolina, Chapel Hill, North Carolina

${ }^{3}$ NoviSci, LLC, Durham, North Carolina

${ }^{4}$ Department of Rheumatology, Park Nicollet Health Services, Minneapolis, Minnesota

${ }^{5}$ Division of Health Policy and Management, School of Public Health, University of Minnesota, Minneapolis, Minnesota

${ }^{6}$ Research Institute, California Pacific Medical Center, San Francisco, California

${ }^{7}$ Bone and Mineral Unit, Oregon Health and Science University, Portland, Oregon

${ }^{8}$ Garvan Institute of Medical Research, UNSW School of Public Health and Community Medicine, New South Wales, Australia; Centre for Health Technologies, University of Technology, Sydney, Australia

${ }^{9}$ Departments of Medicine and Division of Rheumatology, Center for Musculoskeletal Health, UC Davis Health System, Sacramento, California

${ }^{10}$ Department of Family Medicine and Public Health, University of California, San Diego, La Jolla, California

${ }^{11}$ Department of Medicine, University of California, San Diego, La Jolla, California

${ }^{12}$ School of Public Health, Oregon Health and Science University, Portland, Oregon

${ }^{13}$ Department of Medicine, School of Public Health, University of Minnesota, Minneapolis, Minnesota

${ }^{14}$ Division of Epidemiology and Community Health, School of Public Health, University of Minnesota, Minneapolis, Minnesota

Address correspondence to: Margaret L. Gourlay, MD, MPH, UNC Department of Family Medicine, Aycock Building, Manning Drive, CB \#7595, UNC-Chapel Hill, Chapel Hill NC 27599-7595. margaret_gourlay @ med.unc.edu; Telephone: (984)974-4468; Fax: (919)966-6125.

No other financial disclosures were reported by the authors of this paper. 
${ }^{15}$ Center for Chronic Disease Outcomes Research, VA Health Care System, Minneapolis, Minnesota

\section{Abstract}

Purpose-We compared the performance of fracture risk assessment tools in older men, accounting for competing risks including mortality.

Methods-A comparative ROC curve analysis assessed the ability of the QFracture, FRAX® and Garvan fracture risk tools, and femoral neck bone mineral density (BMD) T-score with or without age to identify incident fracture in community-dwelling men aged 65 years or older ( $\mathrm{N}=4994)$ without hip or clinical vertebral fracture or antifracture treatment at baseline.

Results-Among risk tools calculated with BMD, the discriminative ability to identify men with incident hip fracture was similar for FRAX (AUC 0.77, 95\% CI 0.73, 0.81), the Garvan tool (AUC $0.78,95 \%$ CI 0.74, 0.82), age plus femoral neck BMD T-score (AUC 0.79, 95\% CI 0.75, 0.83) and femoral neck BMD T-score alone (AUC 0.76, 95\% CI 0.72, 0.81). Among risk tools calculated without BMD, the discriminative ability to identify hip fracture was similar for QFracture (AUC 0.69, 95\% CI 0.66, 0.73), FRAX (AUC 0.70, 95\% CI 0.66, 0.73) and the Garvan tool (AUC 0.71, $95 \%$ CI $0.67,0.74)$. Correlated ROC curve analyses revealed better diagnostic accuracy for risk scores calculated with BMD compared to QFracture ( $\mathrm{p}$ 0.0001). Calibration was good for the internally generated BMD T-score predictor with or without age and poor for the externally developed risk tools.

Conclusion-In untreated older men without fragility fractures at baseline, an age plus femoral neck BMD T-score classifier identified men with incident hip fracture as accurately as more complicated fracture risk scores.

\section{Keywords}

bone density; fractures; male; osteoporosis; risk assessment

\section{INTRODUCTION}

In the setting of a US Preventive Services Task Force Grade I (insufficient) evidence grade for male osteoporosis screening [1], optimal preventive bone health care is undefined for older men. Fewer good-quality trials of osteoporosis treatment have been conducted in men compared to women, and trials focused on male participants are less likely to have a preventive purpose [2,3]. Male patients and primary care physicians appear to have low awareness of male osteoporosis [4], with typically low rates of bone mineral density (BMD) testing [5]. In the US, Medicare insurance only covers BMD testing in men for a limited number of disease conditions, not including routine screening [6]. While fracture risk assessment tools have been developed for use in osteoporosis screening in primary care settings, uptake in clinical practice is low for male patients, even in high-risk men with secondary causes of BMD loss [7].

While the benefits and risks of routine BMD screening and osteoporosis treatment in men remain uncertain, risk assessment tools available since 2007 may offer a feasible and low- 
cost way to assess fracture risk and potential need for antifracture treatment. Of these, the FRAX ${ }^{\circledR}[8]$, Garvan algorithm [9] and QFracture [10] are the best-studied that can be applied to older men [11], and the reported accuracy of these tools is similar in both sexes [12]. FRAX ${ }^{\circledR}$ was constructed based on primary data from nine population-based cohorts internationally and includes treatment thresholds individualized for 57 countries including the US $[13,14]$. FRAX® incorporates 11 clinical risk factors for a designated country and ethnic group and can be calculated with or without BMD. The Garvan algorithm [15] was developed from 15 years of follow-up data from 858 men and 1358 women aged 60+ years at baseline from the Dubbo Osteoporosis Epidemiology Study in Australia. The Garvan tool incorporates five clinical risk factors and can be calculated with or without BMD. The QFracture tool [16] was developed and internally validated in a UK database comprising 3.7 million patients, contributing 50,755 osteoporotic fractures, 19,531 hip fractures, and 25 million person years. QFracture incorporates 25 clinical risk factors and is calculated without BMD. The tools generate risks of osteoporotic fracture or hip fracture over 10 years (FRAX), 5 or 10 years (Garvan) or 1 to 10 years (QFracture). The FRAX ${ }^{\circledR}$ and Garvan tools have been validated and all three tools have been independently studied more than once in a population-based setting [12]. A 2017 retrospective cohort study using data from external electronic health records from patients aged 50 and older compared all three tools and concluded that QFracture and FRAX had high discriminatory power for hip fracture prediction [17].

Previous diagnostic accuracy analyses of hip fracture prediction have rarely considered the competing risks of clinical vertebral fracture, osteoporosis by BMD criteria, antifracture treatment or death. If individuals develop any of these conditions, risk factor assessment is no longer needed for a treatment decision because treatment is already indicated, in progress, or no longer possible. Methods to account for competing risks avoid inaccurate estimation of the number of fracture events that might be prevented by risk assessment.

We conducted a comparative diagnostic accuracy analysis of the FRAX®, Garvan algorithm and QFracture in a cohort of community-dwelling men aged 65 years and older without hip or clinical vertebral fracture, antifracture treatment or osteoporosis (if BMD was included in the risk score) at baseline, accounting for competing risks that occurred at baseline or during follow-up. We hypothesized that risk scores calculated with BMD would have better discriminatory ability than BMD alone or risk scores calculated without BMD. Based on a 2009 comparison of the FRAX tool to age plus BMD or age plus previous fracture in older women [18], we also hypothesized that simple tools (e.g. BMD T-score with or without age) would perform as accurately as more complicated fracture risk tools in older men.

\section{METHODS}

\section{Setting and Participants}

The Osteoporotic Fractures in Men (MrOS) Study cohort comprised 5994 communitydwelling, ambulatory men aged 65 years and older recruited beginning in March 2000 who received serial dual energy x-ray absorptiometry (DXA) measurements, lateral thoracic and lumbar spine $\mathrm{x}$-rays and fracture follow-up over an average of 15.8 years [19,20]. Participants were also followed every 4 months by postcard or telephone to ascertain 
fractures that were adjudicated by central review of radiology reports [19]. The study protocol and consent documents were approved by Institutional Review Board review committees at six clinical sites in the U.S. (Birmingham, AL; Minneapolis, MN; Palo Alto, CA; Pittsburgh, PA; Portland, OR; San Diego, CA). The plan for the current analysis was approved by the Institutional Review Board at the University of North Carolina.

Men were eligible for the analysis of fracture risk scores calculated with BMD if they did not have a history of hip or clinical vertebral fracture, past or current US Food and Drug Administration-approved antifracture treatment (bisphosphonate, calcitonin or teriparatide), or osteoporosis by BMD criteria at baseline (Online Supplement eFigure 1, eFigure 2). Men were eligible for the analysis of fracture risk scores calculated without BMD if they did not report a previous hip or clinical vertebral fracture or past or current treatment at baseline.

\section{Definition of Osteoporosis}

Osteoporosis was defined according to the 1994 WHO Technical Report [21] as BMD Tscore ([BMD of participant - mean BMD of young reference population]/SD of BMD of reference population) $\leq-2.50$ at the lumbar spine, femoral neck or total hip. Consistent with recommendations from the International Osteoporosis Foundation [22] and International Society for Clinical Densitometry [23], T-scores were calculated using National Health and Nutrition Examination Study III BMD norms for white women aged 20-29 years [24,25].

When the BMD T-score was $>-2.50$ in a given BMD measurement (pre-) and $\leq 2.50$ at the subsequent BMD measurement (post-), the date of "incident" osteoporosis was estimated to be the midpoint of the pre- and post-visits.

\section{Reference and Classification Variables}

The primary reference variable (outcome) was hip fracture and the secondary reference variable was major osteoporotic fracture (clinical spine, forearm, hip or shoulder fracture per FRAX® [14] definition) ascertained during full study follow-up.

The primary classification variable (predictor) was estimated 10-year absolute risk of hip fracture calculated using FRAX® (http://www.shef.ac.uk/FRAX/) or the Garvan algorithm [9] with or without BMD, and without BMD using QFracture [10]. The secondary classification variable was estimated 10-year absolute risk of major osteoporotic fracture calculated using the FRAX ${ }^{\circledR}$ or Garvan algorithm with or without BMD, and without BMD using QFracture. The risk scores were calculated using externally generated parameter coefficients provided by the developers of these algorithms.

In the with-BMD analysis, tertiary classification variables were femoral neck BMD T-score alone, and a variable for age plus femoral neck T-score created using an internally generated logistic regression model. The latter classification variable corresponds to the estimated probability of fracture given age plus femoral neck T-score as predictors with no interaction term. In the without-BMD analysis, continuous age and continuous BMI were tertiary classification variables. 


\section{Accounting for Competing Risks}

To account for competing risks, only men who had a hip fracture or major osteoporotic fracture before developing a competing risk (antifracture treatment, death, or incident osteoporosis for the with-BMD analysis) were considered to be cases. The date of "incident" treatment was estimated to be the midpoint of the pre- (no reported treatment) and postvisits (first reported treatment). Because competing risks cannot be components of an outcome, hip and clinical vertebral fractures were not competing risks in the analysis of incident major osteoporotic fracture. Because the presence of osteoporosis was only known if BMD was measured, osteoporosis was an exclusion criterion and a competing risk in the with-BMD analysis only.

\section{Statistical Analysis}

\section{Correlated ROC Curve Analysis for Identification of Incident fracture-We}

constructed receiver operating characteristic (ROC) curves to determine the area under the curve (AUC) and the corresponding 95\% confidence intervals for each classification variable to identify the reference variable. Each point on the ROC curve marks the sensitivity and corresponding value of " 1 - specificity" for a given cut point of the classification variable. The AUC is an overall estimate of the accuracy of the risk score to identify patients who had a hip or major osteoporotic fracture during follow-up. This area could range from 1 for a perfect test, to 0.5 for a test that performs no better than chance.

For the correlated ROC curve analysis, we used the SAS \%ROC macro based on DeLong's methods for nonparametric comparison of areas under correlated ROC curves, i.e. ROC curves compared within the same population [26,27]. The ROC curves were based on logistic regression to model the observed fracture incidence (reference variable), and estimation of 10-year probability of fracture using the calculated risk scores (classification variables). Separate correlated ROC curve analyses were conducted for the with- and without-BMD scores. Pairwise comparisons of the area under the curve for each classification variable versus the QFracture (referent) were conducted using Chi-square tests with a Bonferroni correction for multiple comparisons, i.e. $P<0.01$ was statistically significant for five pairwise comparisons.

\section{Sensitivity and Specificity to Identify Incident Fracture-We calculated the} sensitivity (true positive fraction), 1 - specificity (false positive fraction) and corresponding 95\% confidence intervals for each classification variable to identify the reference variable. We adjusted risk tool threshold until $90 \%$ sensitivity was reached, then the associated specificity was calculated.

Assessment of Model Calibration-Model calibration (relationship between predicted probability and observed fracture incidence in the study population) of each risk score was assessed using the Hosmer-Lemeshow goodness-of-fit test [28,29]. Using a Bonferroni correction for multiple comparisons, $P<0.01$ was statistically significant with a small $P$ value suggesting poor fit of the predicted fracture risk to the observed fracture incidence.

All analyses were performed using SAS 9.4 [30] or R version 3.3.3 [31]. 


\section{RESULTS}

Baseline characteristics of the 4944 participants in the analytic cohort for the primary analysis (with-BMD analysis for hip fracture) were tabulated (Table 1). The mean age of the with-BMD cohort for hip fracture was 73.4 (5.8) years, and the mean BMI was 27.6 (3.8) $\mathrm{kg} / \mathrm{m}^{2}$ (overweight).

\section{Hip Fracture}

Correlated ROC Curve Analysis for Hip Fracture-Among risk scores calculated with BMD, FRAX (AUC 0.77, 95\% CI 0.73, 0.81) performed similarly to the Garvan tool (AUC 0.78, 95\% CI 0.74, 0.82), age plus femoral neck BMD T-score (AUC 0.79, 95\% CI $0.75,0.83$ ) and BMD T-score alone (AUC $0.76,95 \% \mathrm{CI} 0.72,0.81$ ) to identify men who had incident hip fracture (Table 2; Online Supplement eFigure 3). A correlated ROC curve analysis showed a statistically significant difference between the risk scores calculated with BMD compared to the QFracture that does not include BMD (QFracture AUC 0.69, 95\% CI 0.64, 0.74; $P$ value for comparison with other tools $<0.0001$ ).

Among risk scores calculated without BMD, QFracture (AUC 0.69, 95\% CI 0.66, 0.73) performed similarly to FRAX (AUC 0.70, 95\% CI 0.66, 0.73), the Garvan tool (AUC 0.71, $95 \% \mathrm{CI} 0.67,0.74$ ) and continuous age alone (AUC $0.71,95 \%$ CI $0.66,0.75$ ) to identify men who had incident hip fracture (Table 3; Online Supplement eFigure 4). A correlated ROC curve analysis showed a statistically significant difference between the risk scores calculated without BMD compared to BMD $(P<0.0001$ for comparison with QFracture as referent).

Sensitivity and Specificity for Hip Fracture-The cut points necessary to achieve $90 \%$ sensitivity were less than $2 \%$ for all three risk tools (Tables 2 and 3). Continuous age alone predicted hip fracture risk and major osteoporotic fracture risk with $90 \%$ sensitivity using cut points of 69.5 years (Table 3 ) and 68.5 years (Table 5) respectively.

\section{Major Osteoporotic Fracture}

Correlated ROC Curve Analysis for Major Osteoporotic Fracture-Correlated ROC curve and sensitivity and specificity analyses showed that the tools had similar but slightly lower accuracy to identify major osteoporotic fracture compared to findings for the hip fracture outcome (Tables 4 and 5; Online Supplement eFigure 5, eFigure 6). Among risk scores calculated with BMD, FRAX (AUC $0.72,95 \%$ CI $0.68,0.76$ ) performed similarly to the Garvan tool (AUC 0.74, 95\% CI 0.70, 0.78), and slightly less well than age plus femoral neck BMD T-score (AUC 0.77, 95\% CI 0.73, 0.81) and femoral neck BMD T-score alone (AUC $0.76,95 \%$ CI $0.71,0.80$ ) to identify men who had incident major osteoporotic fracture. A correlated ROC curve analysis showed a statistically significant difference between the risk scores calculated with BMD compared to the QFracture (QFracture AUC 0.65, 95\% CI 0.61, 0.70; P-value for comparison of all tools < 0.0001).

Among risk scores calculated without BMD, QFracture (AUC 0.64, 95\% CI 0.61, 0.68) performed similarly to FRAX (AUC 0.66, 95\% CI 0.62, 0.69), the Garvan tool (AUC 0.66, $95 \% \mathrm{CI} 0.62,0.70$ ) and continuous age alone (AUC $0.67,95 \% \mathrm{CI} 0.62,0.71$ ) to identify 
men who had incident major osteoporotic fracture (Table 3). Continuous BMI performed slightly better than chance to identify incident major osteoporotic fracture (AUC 0.57, 95\% CI $0.53,0.62$ ).

Sensitivity and Specificity for Major Osteoporotic Fracture-The cut points necessary to achieve $90 \%$ sensitivity were less than $7 \%$ for all three risk tools (Tables 4 and 5). Continuous age predicted major osteoporotic fracture risk with $90 \%$ sensitivity using a cut point of 68.5 years.

\section{Model Calibration}

The FRAX, Garvan tool and QFracture were poorly calibrated; calibration plots revealed that the risk scores underestimated observed hip fracture incidence in the lowest deciles of scores and overestimated observed hip fracture incidence in the highest deciles of scores (Figure 1). The age plus femoral neck BMD T-score and femoral neck BMD T-score classification variables developed in the current study showed good calibration to the observed hip fracture incidence rates (Online Supplement eTable 1, eTable 2).

\section{Proportion identified by treatment-level FRAX scores and osteoporosis-level T-score}

In participants who had an incident hip fracture during follow-up: $72.6 \%$ had a treatmentlevel FRAX score (either 10-year risk of hip fracture $23 \%$ or 10-year risk of major osteoporotic fracture $220 \%$ ) before the hip fracture; $99.6 \%$ (271 out of the 272 hip fracture cases) reached age 70 before the hip fracture.

In participants who had an incident major osteoporotic fracture during follow-up: $8.5 \%$ had a treatment-level FRAX score (either 10-year risk of hip fracture $\geq 3 \%$ or 10 -year risk of major osteoporotic fracture $220 \%$ ) before the hip fracture; $98.1 \%$ (638 out of 650 cases) reached age 70 before the major osteoporotic fracture. Also, $82.2 \%$ of all men who developed osteoporosis were age 70 or older and $94.1 \%$ of all men who developed a treatment-level FRAX score (either 10-year risk of hip fracture $\geq 3 \%$ or 10 -year risk of major osteoporotic fracture $\geq 20 \%$ ) were age 70 or older.

\section{Effect of Competing Risks}

Competing risks had minimal impact on the AUCs in the main analysis (to identify hip fracture), but were more influential in the analysis of major osteoporotic fracture. For example, the respective areas under the curve when competing risks were considered vs. not considered were 0.72 versus 0.65 for FRAX with BMD, 0.74 versus 0.68 for the Garvan tool with BMD, 0.65 versus 0.63 for the QFracture, 0.77 versus 0.68 for age plus femoral neck BMD T-score, and 0.76 versus 0.65 for continuous femoral neck T-score to identify major osteoporotic fracture. (Data not shown.)

\section{DISCUSSION}

We conducted a comparative diagnostic accuracy analysis of three fracture risk assessment tools for older men. Our findings suggest that a model based on age plus femoral neck BMD T-score has discrimination similar to more complicated tools for identification of older men 
who will suffer a hip or major osteoporotic fracture. Among risk scores without BMD, all risk scores performed similarly to a model based on continuous age alone. Our findings were similar to past analyses except for weaker performance for QFracture in our study compared to other studies [12]. Continuous age identified hip fracture and major osteoporotic fracture with $90 \%$ sensitivity at a cut point of 70 years, which is recommended in some clinical practice guidelines as a reasonable age to begin BMD screening in older men [32,33].

Ettinger and Ensrud et al. analyzed the diagnostic accuracy of FRAX® to identify hip fracture or major osteoporotic fracture in men aged 65 and older [34]. Accounting for mortality as a competing risk, they found that the FRAX ${ }^{\circledR}$ risk calculator without BMD was better calibrated to hip fracture than to major osteoporotic fracture. With the exception of the Garvan score calculated without BMD, we found poor calibration for risk scores using externally generated model parameters. As expected, our internally generated age plus femoral neck T-score and femoral neck T-score classification variables showed good calibration to the MrOS data. Our results appear to be consistent with a 2015 systematic review in which seven of 15 studies reported poor calibration of at least one fracture risk assessment instrument, with similar levels of calibration for simple (5 or fewer clinical variables) versus more complicated risk tools [35]. Because direct comparison of tools with externally versus internally generated parameters is prone to bias, the predictive ability of simple versus more complex models needs to be confirmed in other cohorts.

A 2014 systematic review and meta-analysis reported satisfactory diagnostic accuracy (AUC>0.70) for 13 different fracture risk assessment tools that had been externally validated, with the best performance seen by QFracture (AUC 0.87, 95\% CI 0.86 to 0.88 for men) [12]. Other analyses of QFracture also showed higher AUC values than were found in our analysis [36,10,17]. The poorer performance of QFracture in our analysis was probably largely due to the older age of our cohort compared to the QFracture development cohort (mean age 73.4 years vs. 50 years respectively). Kanis et al. reported that for hip fracture prediction, validated clinical risk factors for fracture and BMD had better discriminatory ability at younger ages, with the greatest discrepancy for older versus younger cohorts seen for risk scores that incorporated both clinical risk factors and BMD $[37,38]$. Our results may also differ because we excluded men with hip or spine fracture or treatment at baseline to account for competing risks; in contrast, patients with a previous recorded fracture were eligible for inclusion in the QFracture development cohort [36]. Also, risk factors including living in a nursing or care home and chronic liver disease assessed in the QFracture risk score were not present or not measurable in our cohort. Our results demonstrate that fracture risk assessment tools need to be compared within cohorts of the same individuals, as we have done in our correlated ROC curve analyses.

Our results have implications for primary care practice. The National Osteoporosis Foundation clinical practice guidelines recommend risk factor assessment and osteoporosis treatment for older men based on existing evidence supporting vertebral fracture reduction in men treated with bisphosphonates [39,40,32]. Our findings suggest that complicated tools should be reserved for patients for whom the clinician and/or patient himself wishes to obtain additional fracture risk estimates given unique risk factors such as rheumatoid arthritis or oral glucocorticoid use. The poor calibration of the externally generated risk 
scores suggests that recalibrated versions of FRAX, Garvan and QFracture created specifically for adults aged 65 and older would be useful. In the absence of risk scores tailored to an older population, our results suggest that age plus femoral neck BMD T-score is likely to perform as accurately as more complicated risk scores.

Our study had several limitations. The results are not generalizable to postmenopausal women or men under age 65 , individuals with secondary causes of osteoporosis or institutionalized individuals. Because most MrOS participants had good general health at baseline, the results may not be generalizable to men with multiple comorbid health conditions that could influence BMD and fracture. Ninety percent of the sample was white; results might differ for nonwhite individuals. Because of the designated endpoints of the risk tools, we only considered hip fracture and major osteoporotic fractures as outcomes; our results do not apply to clinical fractures at other sites or radiographic vertebral fractures. We included non-traumatic and traumatic fractures to decrease bias due to reporting error for fracture mechanism; the results cannot be generalized to traumatic fractures only. While the QFracture and Garvan tools include a component for falls history, these tools alone are not adequate for full assessment of the falls component of fracture risk in older men. The internal generation of our BMD-T-score classifiers ensured good calibration in this study; the same results might not be seen in other cohorts. Some centers in the United Kingdom use a strategy in which patients with high-risk FRAX without BMD scores are treated and those with intermediate-risk scores are candidates for DXA testing [41]. Our competing risks assumptions are inconsistent with this strategy; therefore, our results do not reflect performance of FRAX when used in this manner.

\section{Conclusions}

Age plus femoral neck BMD T-score predicted incident hip fracture or major osteoporotic fracture as accurately as the Garvan, FRAX and QFracture risk scores calculated with or without BMD in men aged 65 and older. The externally developed risk scores were poorly calibrated to hip fracture incidence in an older male study population. Age 70 or older identified hip fracture and major osteoporotic fracture with $90 \%$ sensitivity.

\section{Supplementary Material}

Refer to Web version on PubMed Central for supplementary material.

\section{Acknowledgments}

We acknowledge Carrie Gartland for her assistance with manuscript preparation.

The project described was funded by grant number R01 AG046294 (Gourlay, Ritter, Overman, Fine), grant number UL1TR001111 from the National Center for Advancing Translational Sciences, and grant number K24 AR048841 (Lane) from the National Institute of Arthritis and Musculoskeletal and Skin Diseases. The work of Dr. Ensrud was supported in part with resources and use of facilities at the Minneapolis VA Medical Center. The Osteoporotic Fractures in Men Study is supported by NIH funding. The following institutes provide support: the National Institute on Aging, the National Institute of Arthritis and Musculoskeletal and Skin Diseases, the National Center for Advancing Translational Sciences, and National Institutes of Health Roadmap for Medical Research under the following grant numbers: U01 AG027810, U01 AG042124, U01 AG042139, U01 AG042140, U01 AG042143, U01 AG042145, U01 AG042168, U01 AR066160, and UL1 TR000128. The content is solely the responsibility of the authors and does not necessarily reflect the official views of the funding agencies. The funders had no role in the 
design and conduct of the study; collection, management, analysis, and interpretation of the data; preparation, review, or approval of the manuscript; or in the decision to submit the manuscript for publication.

Dr. Cawthon reports research grants from GlaxoSmithKline. Dr. Orwoll has received research and consulting support from Merck, Eli Lilly, and Amgen. Dr. Lane has received research and or consulting support from Merck, Amgen, and Regeneron. Dr. Kado has received consultant support from Kalytera Therapeutics, Takeda Pharmaceuticals, and Amgen.

\section{References}

1. Screening for osteoporosis: U.S. preventive services task force recommendation statement. Ann Intern Med. 2011; 154(5):356-364. doi:0003-4819-154-5-201103010-00307 [pii]. DOI: 10.1059/0003-4819-154-5-201103010-00307 [PubMed: 21242341]

2. Zhou J, Wang T, Zhao X, Miller DR, Zhai S. Comparative Efficacy of Bisphosphonates to Prevent Fracture in Men with Osteoporosis: A Systematic Review with Network Meta-Analyses. Rheumatol Ther. 2016; 3(1):117-128. DOI: 10.1007/s40744-016-0030-6 [PubMed: 27747517]

3. Barnard K, Lakey WC, Batch BC, Chiswell K, Tasneem A, Green JB. Recent Clinical Trials in Osteoporosis: A Firm Foundation or Falling Short? PLoS One. 2016; 11(5):e0156068.doi: 10.1371/ journal.pone.0156068 [PubMed: 27191848]

4. Shawa H, Favela E, Diaz J. Knowledge of osteoporosis among men in the primary care setting. South Med J. 2011; 104(8):584-588. DOI: 10.1097/SMJ.0b013e3182241da1 [PubMed: 21886068]

5. Jain S, Bilori B, Gupta A, Spanos P, Singh M. Are Men at High Risk for Osteoporosis Underscreened? A Quality Improvement Project. Perm J. 2016; 20(1):60-64. DOI: 10.7812/TPP/ 14-190 [PubMed: 26824964]

6. Centers for Medicare \& Medicaid Services. [Accessed July 12, 2017] Medicare.gov: the Official U.S. Government Site for Medicare. Your Medicare Coverage: Bone mass measurement (bone density). https://www.medicare.gov/coverage/bone-density.html

7. Damji AN, Bies K, Alibhai SM, Jones JM. Bone health management in men undergoing ADT: examining enablers and barriers to care. Osteoporos Int. 2015; 26(3):951-959. DOI: 10.1007/ s00198-014-2997-6 [PubMed: 25526712]

8. Kanis J, Johnell O, Oden A, Johansson H, McCloskey E. FRAX and the assessment of fracture probability in men and women from the UK. Osteoporos Int. 2008; 19:385-397. [PubMed: 18292978]

9. Nguyen ND, Frost SA, Center JR, Eisman JA, Nguyen TV. Development of a nomogram for individualizing hip fracture risk in men and women. Osteoporos Int. 2007; 18(8):1109-1117. DOI: 10.1007/s00198-007-0362-8 [PubMed: 17370100]

10. Hippisley-Cox J, Coupland C. Predicting risk of osteoporotic fracture in men and women in England and Wales: prospective derivation and validation of QFractureScores. BMJ. 2009; 339:b4229.doi: 10.1136/bmj.b4229 [PubMed: 19926696]

11. Leslie WD, Lix LM. Comparison between various fracture risk assessment tools. Osteoporos Int. 2014; 25(1):1-21. DOI: 10.1007/s00198-013-2409-3 [PubMed: 23797847]

12. Marques A, Ferreira RJ, Santos E, Loza E, Carmona L, da Silva JA. The accuracy of osteoporotic fracture risk prediction tools: a systematic review and meta-analysis. Ann Rheum Dis. 2015; 74(11):1958-1967. DOI: 10.1136/annrheumdis-2015-207907 [PubMed: 26248637]

13. Kanis JA, Oden A, Johansson H, Borgstrom F, Strom O, McCloskey E. FRAX and its applications to clinical practice. Bone. 2009; 44(5):734-743. DOI: 10.1016/j.bone.2009.01.373 [PubMed: 19195497]

14. World Health Organization Collaborating Centre for Metabolic Bone Diseases, University of Sheffield, UK. University of Sheffield; Sheffield, UK: FRAX: WHO Fracture Risk Assessment Tool [Internet]. Available from http://www.shef.ac.uk/FRAX [Accessed September 12, 2017]

15. Nguyen ND, Frost SA, Center JR, Eisman JA, Nguyen TV. Development of prognostic nomograms for individualizing 5-year and 10-year fracture risks. Osteoporosis international : a journal established as result of cooperation between the European Foundation for Osteoporosis and the National Osteoporosis Foundation of the USA. 2008; 19(10):1431-1444. DOI: 10.1007/ s00198-008-0588-0 
16. Collins GS, Mallett S, Altman DG. Predicting risk of osteoporotic and hip fracture in the United Kingdom: prospective independent and external validation of QFractureScores. BMJ. 2011; 342:d3651.doi: 10.1136/bmj.d3651 [PubMed: 21697214]

17. Dagan N, Cohen-Stavi C, Leventer-Roberts M, Balicer RD. External validation and comparison of three prediction tools for risk of osteoporotic fractures using data from population based electronic health records: retrospective cohort study. BMJ. 2017; 356:i6755.doi: 10.1136/bmj.i6755 [PubMed: 28104610]

18. Ensrud KE, Lui LY, Taylor BC, Schousboe JT, Donaldson MG, Fink HA, Cauley JA, Hillier TA, Browner WS, Cummings SR. A comparison of prediction models for fractures in older women: is more better? Arch Intern Med. 2009; 169(22):2087-2094. doi:169/22/2087 [pii]. DOI: 10.1001/ archinternmed.2009.404 [PubMed: 20008691]

19. Orwoll E, Blank JB, Barrett-Connor E, Cauley J, Cummings S, Ensrud K, Lewis C, Cawthon PM, Marcus R, Marshall LM, McGowan J, Phipps K, Sherman S, Stefanick ML, Stone K. Design and baseline characteristics of the osteoporotic fractures in men (MrOS) study--a large observational study of the determinants of fracture in older men. Contemp Clin Trials. 2005; 26(5):569-585. doi:S1551-7144(05)00107-2 [pii]. DOI: 10.1016/j.cct.2005.05.006 [PubMed: 16084776]

20. Blank JB, Cawthon PM, Carrion-Petersen ML, Harper L, Johnson JP, Mitson E, Delay RR. Overview of recruitment for the osteoporotic fractures in men study (MrOS). Contemporary clinical trials. 2005; 26(5):557-568. DOI: 10.1016/j.cct.2005.05.005 [PubMed: 16085466]

21. World Health Organization. WHO Technical report series no. 843. Geneva: World Health Organization; 1994. Assessment of osteoporotic fracture risk and its role in screening for postmenopausal osteoporosis.

22. Hernlund E, Svedbom A, Ivergard M, Compston J, Cooper C, Stenmark J, McCloskey EV, Jonsson B, Kanis JA. Osteoporosis in the European Union: medical management, epidemiology and economic burden. A report prepared in collaboration with the International Osteoporosis Foundation (IOF) and the European Federation of Pharmaceutical Industry Associations (EFPIA). Arch Osteoporos. 2013; 8(1-2):136.doi: 10.1007/s11657-013-0136-1 [PubMed: 24113837]

23. Watts NB, Leslie WD, Foldes AJ, Miller PD. 2013 International Society for Clinical Densitometry Position Development Conference: Task Force on Normative Databases. Journal of clinical densitometry : the official journal of the International Society for Clinical Densitometry. 2013; 16(4):472-481. DOI: 10.1016/j.jocd.2013.08.001 [PubMed: 24076161]

24. Looker A, Wahner H, Dunn W, Calvo M, Harris T, Heyse S, Johnston C Jr, Lindsay R. Updated data on proximal femur bone mineral levels of US adults. Osteoporos Int. 1998; 8:468-489. [PubMed: 9850356]

25. Looker AC, Melton LJ 3, Borrud LG, Shepherd JA. Lumbar spine bone mineral density in US adults: demographic patterns and relationship with femur neck skeletal status. Osteoporos Int. 2012; 23(4):1351-1360. DOI: 10.1007/s00198-011-1693-z [PubMed: 21720893]

26. DeLong ER, DeLong DM, Clarke-Pearson DL. Comparing the areas under two or more correlated receiver operating characteristic curves: a nonparametric approach. Biometrics. 1988; 44(3):837845. [PubMed: 3203132]

27. SAS Knowledge Base / Samples \& SAS Notes. [Accessed September 12, 2017] Sample 25017: Nonparametric comparison of areas under correlated ROC curves. http://support.sas.com/kb/ 25/017.html

28. Hosmer DW, Lemeshow S. A goodness-of-fit test for the multiple logistic regression model. Communications in Statistics: Theory and Methods. 1980; A10:1043-1069.

29. Hosmer DW, Hosmer T, Le Cessie S, Lemeshow S. A comparison of goodness-of-fit tests for the logistic regression model. Stat Med. 1997; 16(9):965-980. [PubMed: 9160492]

30. SAS Institute Inc. SAS/STAT 9.3 User's Guide. Cary, NC: SAS Institute Inc; 2011.

31. R Core Team. R: A language and environment for statistical computing. R Foundation for Statistical Computing; Vienna, Austria: 2017. URL: https:/www.R-project.org/

32. Cosman F, de Beur SJ, LeBoff MS, Lewiecki EM, Tanner B, Randall S, Lindsay R. National Osteoporosis F. Clinician's Guide to Prevention and Treatment of Osteoporosis. Osteoporos Int. 2014; 25(10):2359-2381. DOI: 10.1007/s00198-014-2794-2 [PubMed: 25182228] 
33. Watts NB, Adler RA, Bilezikian JP, Drake MT, Eastell R, Orwoll ES, Finkelstein JS. Osteoporosis in men: an Endocrine Society clinical practice guideline. J Clin Endocrinol Metab. 2012; 97(6): 1802-1822. DOI: 10.1210/jc.2011-3045 [PubMed: 22675062]

34. Ettinger B, Ensrud KE, Blackwell T, Curtis JR, Lapidus JA, Orwoll ES. Osteoporotic Fracture in Men Study Research G. Performance of FRAX in a cohort of community-dwelling, ambulatory older men: the Osteoporotic Fractures in Men (MrOS) study. Osteoporos Int. 2013; 24(4):11851193. DOI: 10.1007/s00198-012-2215-3 [PubMed: 23179575]

35. Nayak S, Edwards DL, Saleh AA, Greenspan SL. Systematic review and meta-analysis of the performance of clinical risk assessment instruments for screening for osteoporosis or low bone density. Osteoporos Int. 2015; 26(5):1543-1554. DOI: 10.1007/s00198-015-3025-1 [PubMed: 25644147]

36. Hippisley-Cox J, Coupland C. Derivation and validation of updated QFracture algorithm to predict risk of osteoporotic fracture in primary care in the United Kingdom: prospective open cohort study. BMJ. 2012; 344:e3427.doi: 10.1136/bmj.e3427 [PubMed: 22619194]

37. Kanis J, Oden A, Johnell O, Johansson H, DeLaet C, Brown J, Burckhardt P, Cooper C, Christiansen C, Cummings S, Eisman J, Fujiwara S, Gluer C, Goltzman D, Hans D, Krieg M-A, LaCroix A, McCloskey E, Mellstrom D, Melton LJ 3, Pols H, Reeve J, Sanders K, Schott A, Silman A, Torgerson D, van Staa T, Watts N, Yoshimura N. The use of clinical risk factors enhances the performance of BMD in the prediction of hip and osteoporotic fractures in men and women. Osteoporos Int. 2007; 18:1033-1046. [PubMed: 17323110]

38. Browner WS. Predicting fracture risk: Tougher than it looks. IBMS BoneKEy. 2007; 4(8):226-230.

39. Boonen S, Reginster JY, Kaufman JM, Lippuner K, Zanchetta J, Langdahl B, Rizzoli R, Lipschitz S, Dimai HP, Witvrouw R, Eriksen E, Brixen K, Russo L, Claessens F, Papanastasiou P, Antunez O, Su G, Bucci-Rechtweg C, Hruska J, Incera E, Vanderschueren D, Orwoll E. Fracture risk and zoledronic acid therapy in men with osteoporosis. The New England journal of medicine. 2012; 367(18):1714-1723. DOI: 10.1056/NEJMoa1204061 [PubMed: 23113482]

40. Zhong ZM, Chen JT. Anti-fracture efficacy of risedronic acid in men: A meta-analysis of randomized controlled trials. Clin Drug Investig. 2009; 29(5):349-357. DOI: 10.2165/00044011-200929050-00007

41. Compston J, Cooper A, Cooper C, Gittoes N, Gregson C, Harvey N, Hope S, Kanis JA, McCloskey EV, Poole KES, Reid DM, Selby P, Thompson F, Thurston A, Vine N. National Osteoporosis Guideline G. UK clinical guideline for the prevention and treatment of osteoporosis. Arch Osteoporos. 2017; 12(1):43.doi: 10.1007/s11657-017-0324-5 [PubMed: 28425085] 
Hip fracture outcome in with-BMD cohort

Externally generated tools and internally generated BMD T-score -/+ age

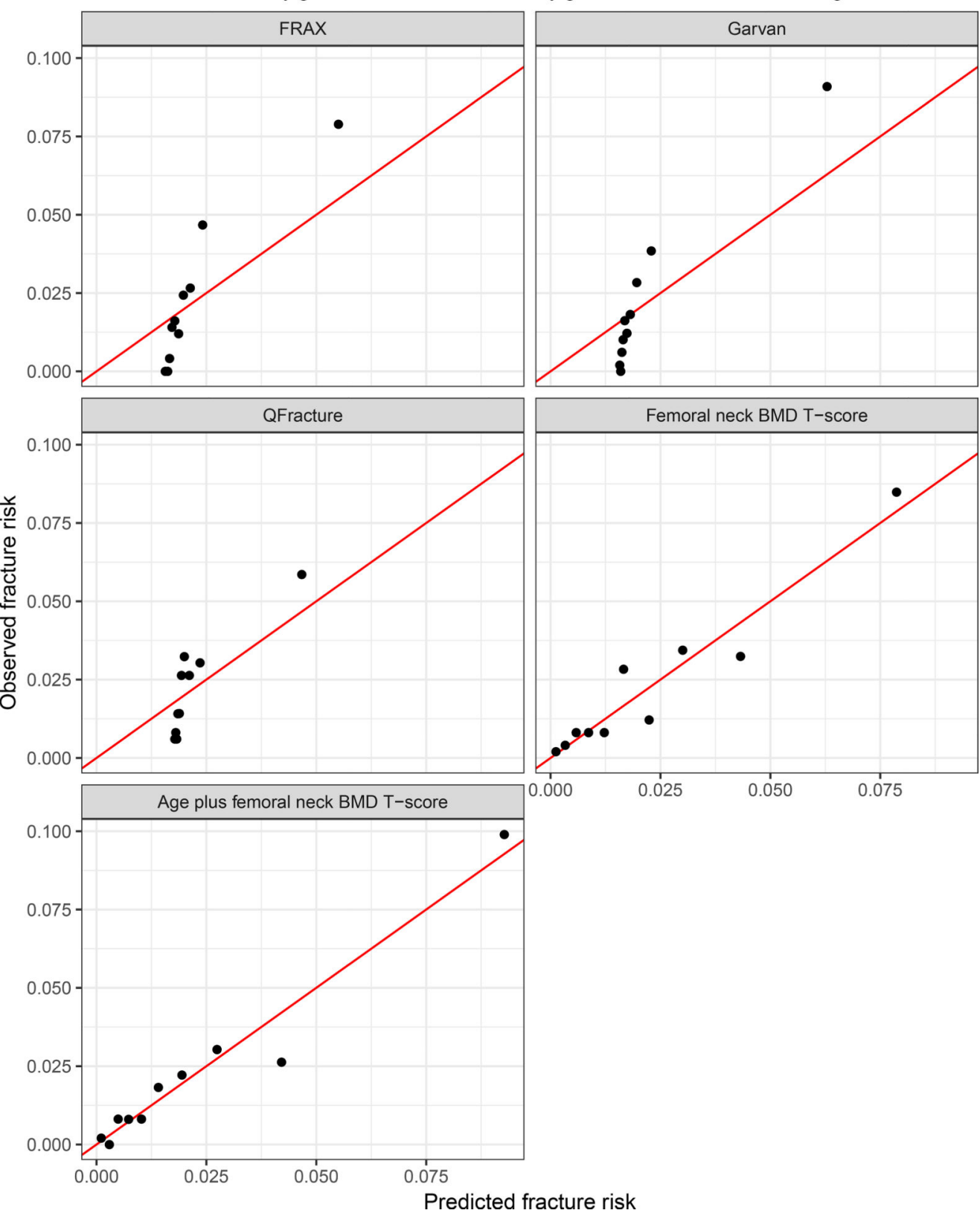

Figure 1.

Arch Osteoporos. Author manuscript; available in PMC 2018 October 20. 


\section{Table 1}

Characteristics of the analytical cohorts

\begin{tabular}{|c|c|c|c|}
\hline Characteristics & $\begin{array}{c}\text { With BMD }{ }^{a} \\
4944(82.5 \%)\end{array}$ & $\begin{array}{l}\text { Without BMD } \\
5200(86.77 \%)\end{array}$ & $\underset{5993}{\operatorname{All~} \operatorname{MrOS}^{b}}$ \\
\hline \multicolumn{4}{|l|}{ Age, mean years (SD) } \\
\hline $65-69$ & $67.1(1.4)$ & $67.1(1.4)$ & $67.1(1.4)$ \\
\hline $70-74$ & $71.9(1.4)$ & $71.9(1.4)$ & $71.9(1.4)$ \\
\hline $75-79$ & $76.7(1.4)$ & $76.8(1.4)$ & $76.8(1.4)$ \\
\hline$\geq 80$ & $83(2.9)$ & $83(2.9)$ & $83(2.9)$ \\
\hline Weight, mean kg (SD) & $84.1(13.1)$ & $83.8(13.3)$ & $83.1(13.3)$ \\
\hline \multicolumn{4}{|l|}{ BMI, kg/m2 } \\
\hline$<25$ & $23.3(1.4)$ & $23.3(1.4)$ & $23.2(1.4)$ \\
\hline$\geq 25$ & $29(3.2)$ & $29(3.2)$ & $29(3.2)$ \\
\hline \multicolumn{4}{|l|}{ Race } \\
\hline White (Non-Hispanic) & $4399(88.98)$ & $4637(89.17)$ & $5361(89.45)$ \\
\hline African American & $221(4.47)$ & 227 (4.37) & $244(4.07)$ \\
\hline Asian & $163(3.3)$ & $170(3.27)$ & $191(3.19)$ \\
\hline Hispanic & $107(2.16)$ & $108(2.08)$ & $126(2.1)$ \\
\hline Other & $54(1.09)$ & $58(1.12)$ & $71(1.18)$ \\
\hline \multicolumn{4}{|l|}{ Years of education } \\
\hline Less than high school & $340(6.88)$ & $357(6.87)$ & $393(6.56)$ \\
\hline High school/Some college & $1997(40.39)$ & $2103(40.44)$ & $2413(40.26)$ \\
\hline College/Some grad school & $1427(28.86)$ & $1497(28.79)$ & $1726(28.8)$ \\
\hline Grad school & $1180(23.87)$ & $1243(23.9)$ & $1461(24.38)$ \\
\hline \multicolumn{4}{|c|}{ Previous fracture after age 50} \\
\hline Yes & 925 (18.71) & $996(19.15)$ & $1360(22.69)$ \\
\hline No & $4019(81.29)$ & $4204(80.85)$ & $4633(77.31)$ \\
\hline \multicolumn{4}{|l|}{ Current smoker } \\
\hline Current & $169(3.42)$ & $184(3.54)$ & $206(3.44)$ \\
\hline Past & $2916(58.98)$ & 3047 (58.6) & $3538(59.04)$ \\
\hline Never & $1858(37.58)$ & $1968(37.85)$ & $2248(37.51)$ \\
\hline Missing & $1(0.02)$ & $1(0.02)$ & $1(0.02)$ \\
\hline \multicolumn{4}{|l|}{ Alcohol consumption } \\
\hline No use & $1723(34.85)$ & $1831(35.21)$ & $2121(35.39)$ \\
\hline$<21$ drinks per week & $3015(60.98)$ & $3153(60.63)$ & $3627(60.52)$ \\
\hline$\geq 21$ drinks per week & $200(4.05)$ & $210(4.04)$ & $237(3.95)$ \\
\hline Don't know or refused & $6(0.12)$ & $6(0.12)$ & $8(0.13)$ \\
\hline
\end{tabular}

History of parental hip fracture 


\begin{tabular}{|c|c|c|c|}
\hline Characteristics & $\begin{array}{c}\text { With BMD } \\
4944(82.5 \%)\end{array}$ & $\begin{array}{l}\text { Without BMD }{ }^{a} \\
5200(86.77 \%)\end{array}$ & 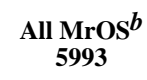 \\
\hline Yes & $602(12.18)$ & $642(12.35)$ & $761(12.7)$ \\
\hline No & $1748(35.36)$ & $1839(35.37)$ & $2126(35.47)$ \\
\hline Don't know & $1146(23.18)$ & $1203(23.13)$ & $1389(23.18)$ \\
\hline Missing & $1448(29.29)$ & $1516(29.15)$ & $1717(28.65)$ \\
\hline \multicolumn{4}{|l|}{ Rheumatoid arthritis } \\
\hline Yes & $249(5.04)$ & $259(4.98)$ & $315(5.26)$ \\
\hline No & $2051(41.48)$ & $2161(41.56)$ & $2532(42.25)$ \\
\hline Missing & $2644(53.48)$ & $2780(53.46)$ & $3146(52.49)$ \\
\hline \multicolumn{4}{|l|}{ Oral glucocorticoid ever-use } \\
\hline Yes & $90(1.82)$ & $97(1.87)$ & $142(2.37)$ \\
\hline No & $3802(76.9)$ & $3976(76.46)$ & $4591(76.61)$ \\
\hline Missing & $1052(21.28)$ & $1127(21.67)$ & $1260(21.02)$ \\
\hline \multicolumn{4}{|c|}{ Hip or major osteoporotic fracture by study end } \\
\hline Hip fracture & $175(3.54)$ & $218(4.19)$ & $272(4.54)$ \\
\hline Major osteoporotic fracture & $326(6.59)$ & $387(7.44)$ & $650(10.85)$ \\
\hline \multicolumn{4}{|c|}{ Antifracture treatment by study end } \\
\hline Bisphosphonate & $5(0.1)$ & $8(0.15)$ & $448(7.48)$ \\
\hline Calcitonin & 0 & 0 & $13(0.22)$ \\
\hline Teriparatide & $1(0.02)$ & $1(0.02)$ & $4(0.07)$ \\
\hline
\end{tabular}

BMD, bone mineral density

${ }^{a}$ Tabulations shown for primary (hip fracture reference variable) with-BMD and without-BMD analyses, accounting for competing risks

${ }^{b}$ One man excluded for age $<65$ 




Arch Osteoporos. Author manuscript; available in PMC 2018 October 20. 


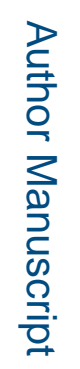

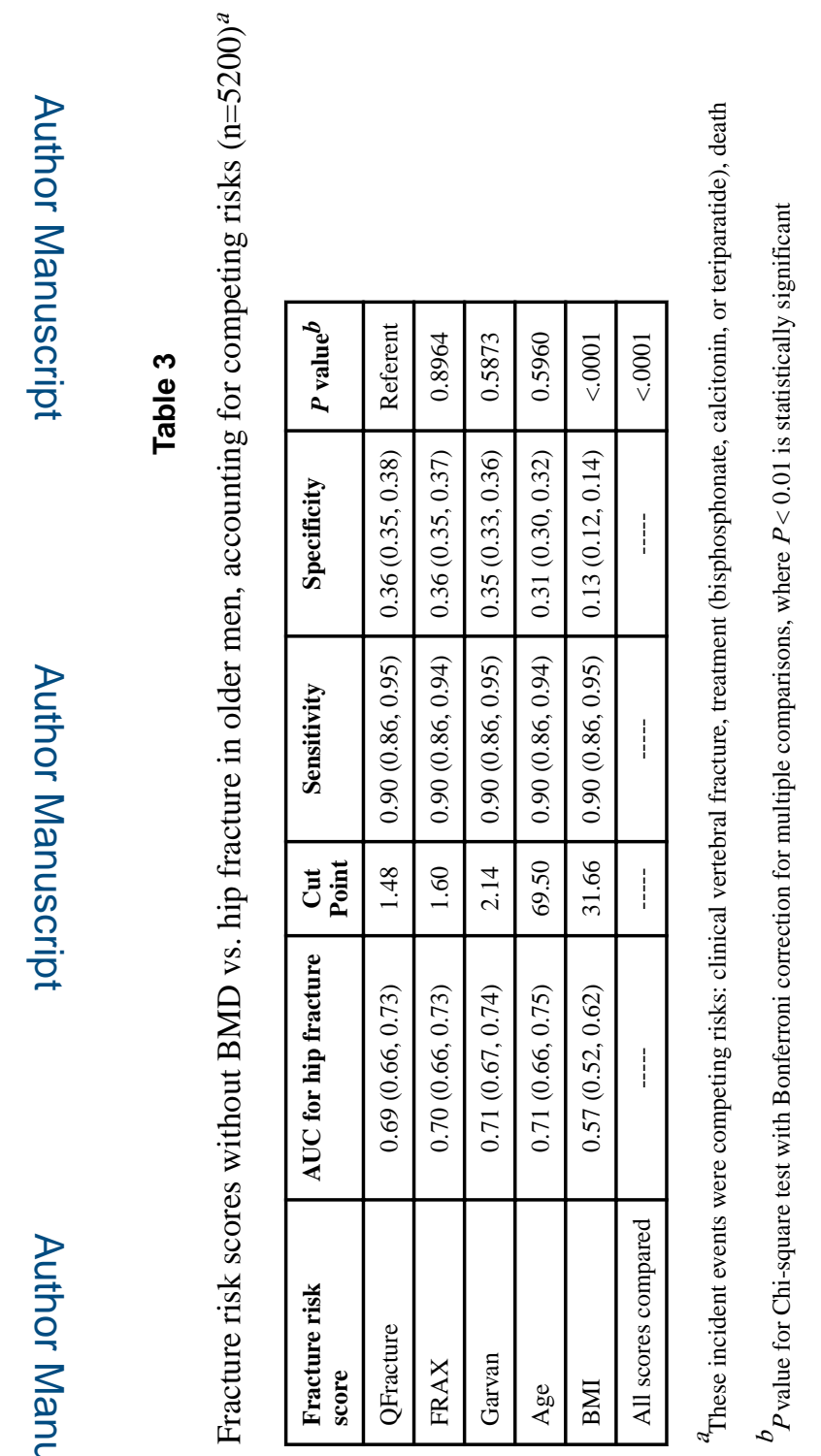

Arch Osteoporos. Author manuscript; available in PMC 2018 October 20. 

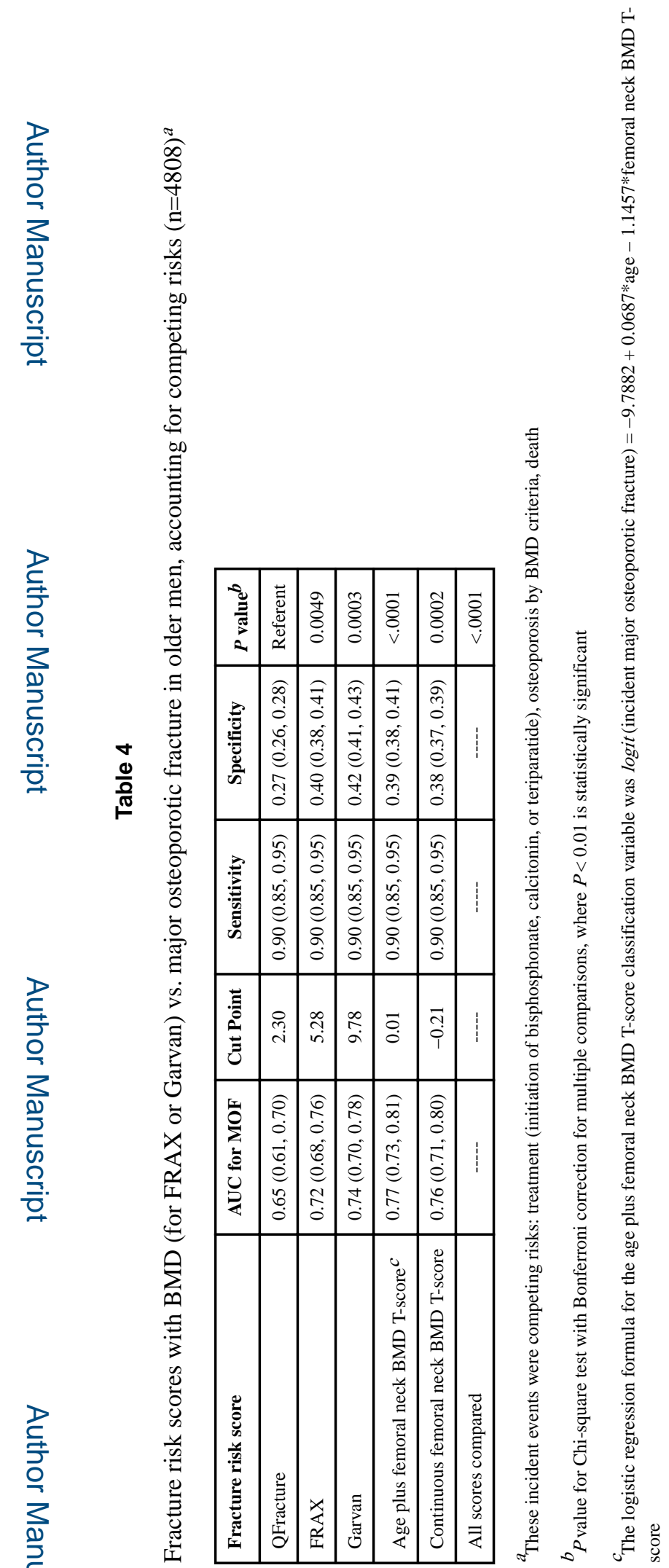

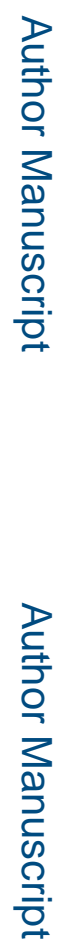

Arch Osteoporos. Author manuscript; available in PMC 2018 October 20. 
로을

로을

กู



Arch Osteoporos. Author manuscript; available in PMC 2018 October 20. 